\title{
NANO-INDENTATION OF COPPER THIN FILMS ON SILICON SUBSTRATES
}

\author{
S. Suresh ${ }^{1}$, T.-G. Nieh ${ }^{2}$ and B.W. Choi ${ }^{2}$ \\ ${ }^{1}$ Department of Materials Science and Engineering, Massachusetts Institute of Technology, \\ Cambridge, MA 02139-4307 U.S.A. ${ }^{2}$ Lawrence Livermore National Laboratory, 7000 East Ave., \\ P.O. Box 808, L-350, Livermore, CA 94551-9900 U.S.A.
}

(Received July 26, 1999)

(Accepted in revised form August 6, 1999)

Keywords: Mechanical properties; Nano-indentation; Thin films; Copper; Dislocations

\section{$\underline{\text { Introduction }}$}

Indentation methods are finding increasing use in the study of mechanical properties of bulk and thin-film materials over a wide range of size scales (e.g., [1,2]). With the increasing sophistication of instrumented, depth-sensing indentation equipment and of computational methods to model the deformation of materials subjected to indentation, there is growing interest in studying the elastoplastic properties and residual stresses in thin films on substrates (e.g., [2,3]) using instrumented indentation.

Nano-indentation studies of thin films provide the possibility of examining a variety of micromechanistic processes using different indentor tip geometries and size scales. Although uncertainties exist, there is a rapidly expanding theoretical and computational effort aimed at providing better interpretations of the elastic modulus, yield strength, strain hardening and internal stresses as well as deformation mechanisms in thin films by recourse to nano-indentation [1-3]. Furthermore, there is experimental evidence from indented bulk $\mathrm{Fe}-\mathrm{Si}, \mathrm{Au}$ and $\mathrm{W}$ single crystals that indentation methods could provide quantitative information about the nucleation of defects, which are ostensibly manifested as abrupt bursts in the penetration depth of the nano-indentor into the material [4-6]. Systematic studies of the mechanical response of thin films to indentation, however, have not been undertaken where the effects of film thickness, texture and grain size on the mechanisms of indentation-induced deformation, including defect nucleation processes, are examined.

We present here experimental results on the nano-indentation of polycrystalline $\mathrm{Cu}$ thin films, of three different thicknesses but with approximately the same grain size and texture, on Si substrates. Care was taken to ensure that the indentation response was solely that of the film, with the deformation field of indentation confined fully within the film thickness, and that the results interpreted from such experiments are not influenced by the specific tip radius of the indentor. Copper was chosen as the material for the thin film in view of its recent emergence as the interconnect material of choice in microelectronic devices. 


\section{$\underline{\text { Materials and Experimental Methods }}$}

Nano-indentation experiments were conducted on copper films, approximately 300, 600 and $1000 \mathrm{~nm}$ in thickness, which were electron-beam-deposited at $50^{\circ} \mathrm{C}$ on to oxidized (100) Si substrates. The films were then vacuum-annealed at $475^{\circ} \mathrm{C}$ for $1 \mathrm{~h}$. The resulting polycrystalline microstructure comprised grains with an average size of approximately $500 \mathrm{~nm}$. Nearly $60-80 \%$ of the grains had (111) texture in the out-of-plane, film-thickness direction, with the rest exhibiting random orientation; the thickness direction of the film typically contained one to two grains.

Nano-indentation experiments were conducted using a commercial Nanoindentor $\mathrm{XP}^{\mathrm{TM}}$ (MTS Corporation, St. Paul, MN). Load-controlled experiments were carried out using the Dynamic Contact Module $\left(\mathrm{DCM}^{\mathrm{TM}}\right)$, which was placed in series with the load train. This facilitated better load resolution, particularly at smaller penetration depths. A diamond Berkovich pyramid indentor with a tip radius, $R \approx$ $50 \mathrm{~nm}$, was used. It is known from nano-indentation tests on metals (e.g., [7]) that the relationship between the indentation load, $P$, and the depth of penetration, $h$, of the indentor into the copper thin film, is influenced by the tip radius $R$ of the nominally 'sharp' Berkovich indentor only in the early stages of elastic deformation, where

$$
P=\frac{4}{3} E^{*} \cdot h^{3 / 2} \cdot R^{1 / 2}, \quad E^{*}=\left\{\frac{1-v_{\mathrm{f}}^{2}}{E_{\mathrm{f}}}+\frac{1-v_{\mathrm{ind}}^{2}}{E_{\mathrm{ind}}}\right\}^{-1},
$$

where $E$ and $\nu$ are Young's modulus and Poisson's ratio, respectively, and the subscripts, 'f' and 'ind' refer to the film and the indentor, respectively. In the present experiments, the copper films comprise a mixture of (111) and randomly oriented grains. Young's moduli values based on Voigt polycrystalline average (representing iso-strain deformation among grains), Reuss average (representing iso-stress deformation), and random grain orientation are 145, 110 and $128 \mathrm{GPa}$, respectively. In view of the relatively small spread between the bounding values of the moduli, the following elastic properties are used in this paper: Young's modulus, $E_{\mathrm{f}}=126 \mathrm{GPa}, \nu_{\mathrm{f}}=0.33, E_{\text {ind }}=1000 \mathrm{GPa}, \nu_{\text {ind }}=0.07$, and $E^{*}$ $\approx 123 \mathrm{GPa}$. As seen later, these values rationalize the elastic deformation response seen in all the $\mathrm{Cu}$ films reasonably well. Analyses have shown that a Berkovich tip radius of $50 \mathrm{~nm}$ influences elastic deformation only to a penetration depth of approximately $5 \mathrm{~nm}$ [7], during which the response is similar to that of a spherical indentor of radius $50 \mathrm{~nm}$. Beyond this penetration depth, however, the elastic response is adequately captured by the trend expected for a sharp Berkovich indentor [8], for which

$$
P=2.189 E^{*} h^{2}\left\{1.00-0.21 v_{\mathrm{f}}-0.01 v_{\mathrm{f}}^{2}-0.41 v_{\mathrm{f}}^{3}\right\} .
$$

For the copper thin films, the elastic response during Berkovich indentation is expected to be captured by Eq. (2) beyond a penetration depth, $h$, of only about $5 \mathrm{~nm}$. Nano-indentation experiments were conducted in such a manner that the penetration depth was significantly greater than this critical value in order to employ the analysis for the sharp Berkovich indentor. At the same time, the maximum depth of penetration of the indentor into the film was designed to be always less than $40 \mathrm{~nm}$ for the $300 \mathrm{~nm}$ thick copper film, and $45 \mathrm{~nm}$ for the 600 and $1000 \mathrm{~nm}$ thick films in order to prevent possible effects of the substrate on the indentation response.

\section{$\underline{\text { Results and Discussion }}$}

\section{$\underline{\text { Experimental Results }}$}

Figures $1(a),(b)$ and $(c)$ show the $P-h$ curves obtained from the nano-indentation of 1000,600 and 300 $\mathrm{nm}$ thick copper films, respectively, on Si substrates. It is seen that each of these curves exhibits a 

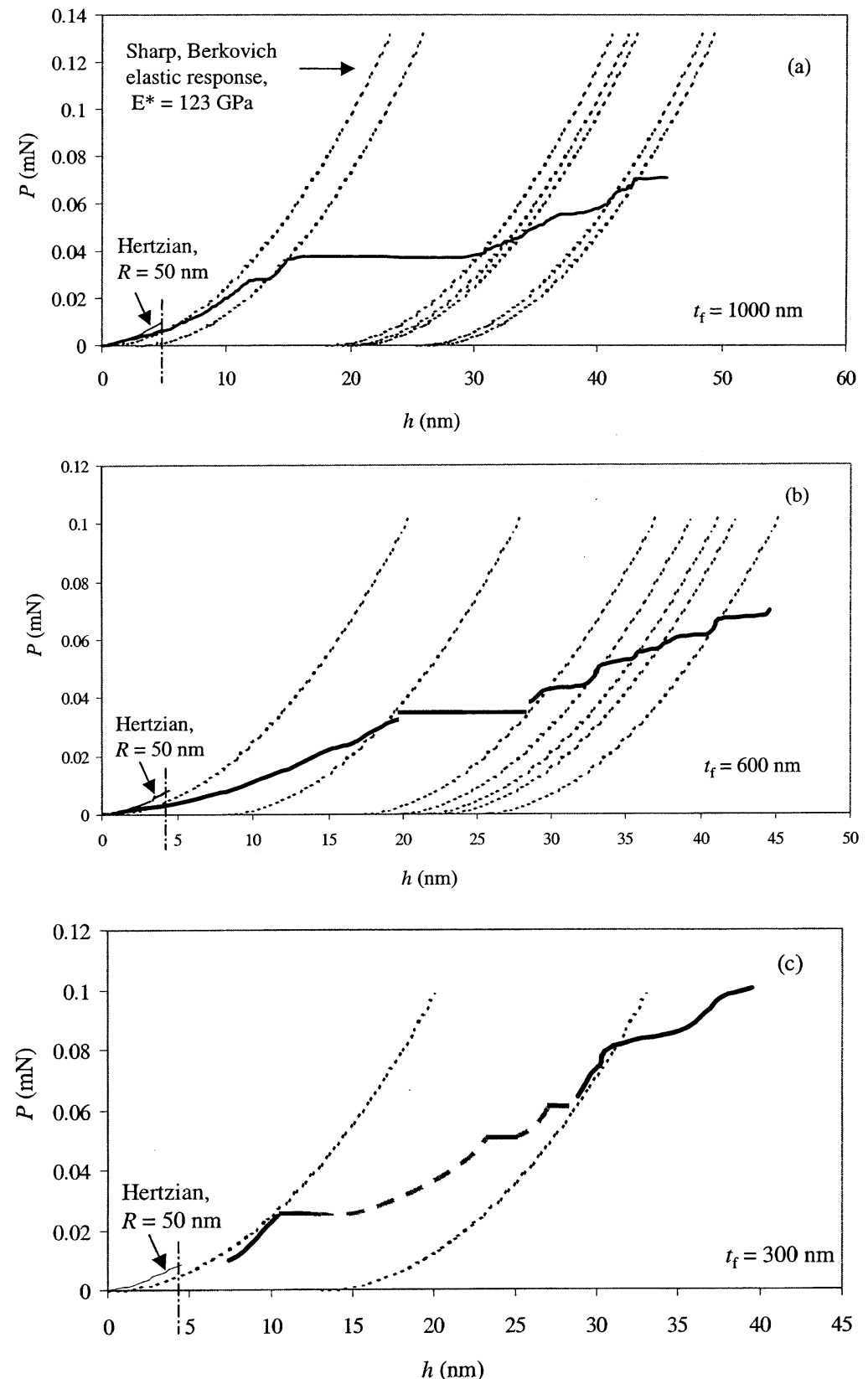

Figure 1. Nano-indentation force versus penetration depth curves for copper thin films of thickness (a) $1000 \mathrm{~nm}$, (b) $600 \mathrm{~nm}$ and (c) $300 \mathrm{~nm}$. The dashed lines denote predicted elastic response for the copper film indented by the sharp Berkovich indentor. The broken segments in the $P-h$ curve in (c) denote regions of experimental uncertainty.

number of discrete 'bursts' in penetration depth, $h$, at different indentation loads; each burst occurs at an approximately constant load. The first burst, which occurs at a load of $35 \pm 10 \mu \mathrm{N}$ (based on observations from a large number of repeat indentations), is approximately independent of film thickness, $t_{\mathrm{f}}$. The number of displacement bursts and the abrupt change in displacement within each 

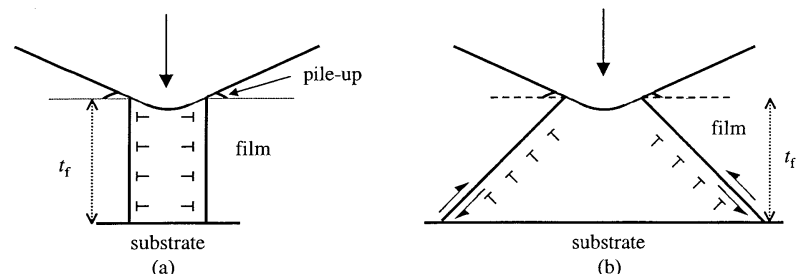

(a)

(b)

Figure 2. Possible mechanisms for the occurrence of dislocation bursts due to (a) nucleation of dislocation loops and (b) shear band formation at the tip of the indentor.

burst, however, are functions of $t_{\mathrm{f}}$; with increasing $t_{\mathrm{f}}$, the $P-h$ curve tends to move further to the right, indicating a greater indentation compliance. In the present experiments, the 'flatness' of the load during the bursts is somewhat reduced for the smallest value of $t_{\mathrm{f}}$.

Figures $1(a)-(c)$ also reveal that, in-between the displacement bursts, the indentation response is determined solely by the elastic properties of the film (for a fixed indentor). The dashed lines in these figures are the elastic indentation responses, predicted for the sharp Berkovich indentor using Eq. (2), by choosing $E^{*}$ to be $123 \mathrm{GPa}$; here, the displacements for different dashed curves are adjusted so as to make the dashed lines coincide with the experimental $P-h$ plot. It is readily seen that the overall indentation behavior of copper thin films comprises two parts: an elastic deformation which is well predicted from a knowledge of only the elastic properties of the film using Eq. (2), and an intermittent, inelastic regime which is characterized by periodic, discrete jumps in indentor displacement into the film. The initial elastic response occurring prior to the first displacement burst (for $h \leq 5 \mathrm{~nm}$ ) is expected to be influenced by the rounded tip of the indentor as well as by the possible presence of any oxide films on the indented surface.

\section{Possible Mechanisms}

Several mechanisms have been postulated for displacement bursts during the nano-indentation of bulk single crystalline materials. These include: (a) the presence of a native oxide at the surface, whose brittle fracture under the indentor triggers an abrupt 'sinking in' of the indentor into the indented material (e.g., [5,6]), and (b) the punching of dislocation loops under the indentor at a critical indentation load $[4,7]$. An examination of the results shown in Fig. 1, along with similar experimental results obtained for monocrystalline and polycrystalline $\mathrm{Al}$ thin films on Si substrates [7], reveals that dislocation nucleation directly beneath the indentor tip is the more probable mechanism for the onset of displacement bursts in thin films during nano-indentation for the following reasons. (1) Multiple displacement bursts are observed in thin films even at indentor penetration depths significantly larger than those corresponding to the thickness of an oxide layer on the surface (of the order of 5-10 nm). (2) The indentation response between two consecutive bursts is elastic for all three film thicknesses, and can be quantified solely from the elastic properties of the film using Eq. (2), as indicated by the dashed curves in Figs. 1(a)-(c). (3) Nano-indentation experiments conducted on thin Au layers without oxide films also exhibit similar displacement bursts [4]. Transmission electron microscopy observations of indented Au layers have led to postulates of the nucleation of geometrically necessary dislocation loops [9].

The mechanisms governing the nucleation of dislocations during nano-indentation are not fully understood. One could, however, envision two possible scenarios for microplastic deformation under the indentor, which are schematically sketched in Fig. 2. Figure 2(a) reveals the punching of prismatic dislocation loops as the indentor periodically sinks into the thin film causing a burst of displacement in the $P-h$ curve. The conservation of volume in the thin film during such microplastic deformation results 
in significant pile-up of material around the indentor which is seen in the present work on Cu thin films, as well as atomic force microscopy (AFM) of nano-indentation response in Al thin films on $\mathrm{Si}$ substrates [7]. The maximum burst in displacement, $\Delta h_{\max }$, occurs in the case of the $1000 \mathrm{~nm}$ film, where the jump in displacement is as much as $15 \mathrm{~nm}$; see the displacement burst from about $15 \mathrm{~nm}$ to $30 \mathrm{~nm}$ in Fig. 1(a). Assuming that this displacement discontinuity is fully accommodated by the nucleation of prismatic dislocation loops, the number of dislocation loops is estimated to be: $n=$ $\Delta h_{\text {max }} / b$, where $b$ is the magnitude of the Burgers vector. Taking $b \approx 0.3 \mathrm{~nm}$, the number of dislocation loops, $n$, nucleated under the indentor is estimated to be approximately 50 . Figure $2(b)$ shows shear band nucleation at the indentor tip. If such a mechanism were to operate, one may envision the possible occurrence of shear localization, somewhat analogous to that seen at the tip of a crack. The ensuing motion of dislocations along the shear bands, which are likely to terminate at a grain boundary or the film-substrate interface, would then be expected to be similar to that of the sliding of a mode II crack. Identification of the more dominant of the two mechanisms in Fig. 2 requires additional microscopic observational evidence.

\section{Possible Condition for the Onset of Displacement Bursts}

It is also of interest to examine possible connections between the mechanics of indentation and the load at which the first departure from elastic deformation occurs during nano-indentation of $\mathrm{Cu}$ thin films. For the nominally sharp Berkovich indentor with a tip radius, $R \approx 50 \mathrm{~nm}$, the maximum shear stress under the sharp Berkovich indentor is [8]: $\tau_{\max } \approx 0.17 E^{*}$, which is approximately $20.88 \mathrm{GPa}$ for the $\mathrm{Cu}$ film. Note that for a fixed indentor geometry and $E^{*}, \tau_{\max }$ is a constant for elastic deformation in the film, and is independent of the applied load. The theoretical shear strength, $\tau_{\text {th }}$, of $\mathrm{Cu}$ may be approximated as $G / 2 \pi=12.7 \mathrm{GPa}$, where $G$ is shear modulus, which is also a constant. If the first departure from elastic deformation during nano-indentation were to occur when $\tau_{\max }$ approached $\tau_{\text {th }}$, this critical condition would be expected to be independent of film thickness. Consistent with this expectation, it is seen in the present case that $\tau_{\max } \sim \tau_{\text {th }}$, and that the first departure from linearity occurs roughly at the same load for all three film thicknesses (see Fig. 1). Similar trends are seen in nano-indented Al thin films [7].

The trends described by Figs. 1(a)-(c) and their implications are schematically summarized in Fig. 3. Figure 3(a) shows typical $P-h$ plot with discrete bursts in indentor penetration depth which are separated by an elastic indentation response. This elastic deformation between the bursts conforms to Kick's law for a sharp indentor, $P=C h^{2}$, where $C$ is a measure of indentation compliance. From Eq. (2), $C$ can be determined uniquely with knowledge of the elastic properties of the film provided that the parabolic relationship between $P$ and $h$ holds. Departure from this Kick's law behavior is seen primarily in the early stages of indentation, for penetration depths typically below $5 \mathrm{~nm}$, where the roundedness of the indentor tip and/or the presence of an oxide scale at the surface can influence the indentation compliance. The overall elastoplastic response of the thin film to nano-indentation can be estimated by considering the bound to the $P-h$ curve which is constructed by linking the extreme right points of each displacement burst, as shown in Fig. 3(b). This consolidated measure of an average 'elastoplastic' indentation stiffness actually comprises the macroscopic, linear elastic response of the film as well as the microscopic plastic deformation arising from the nucleation of dislocations during nano-indentation. Figure 4 shows such consolidated elastoplastic curves, approximated for thin-film and bulk copper. An increase in film thickness leads to a more-compliant response to nano-indentation, thereby indicating that the overall yield strength of the thin film decreases with increasing film thickness. This observation is fully consistent with curvature measurements for thin films on substrates subjected to thermal cycling where the resistance to plastic flow is seen to be lower for higher film thicknesses (e.g., [10]). More direct comparisons of the yield properties of thin films from the present nano-indentation tests with 


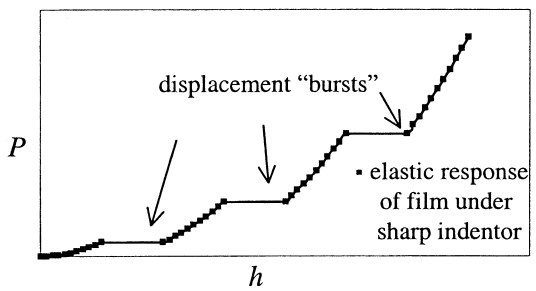

(a)

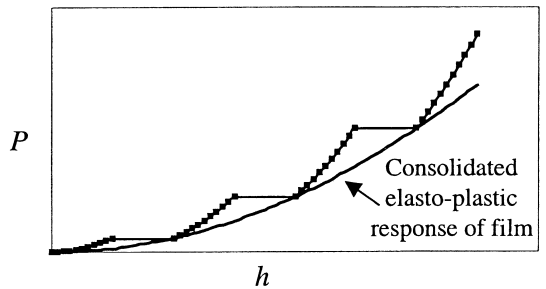

(b)

Figure 3. Schematic of (a) elastic deformation separating the displacement bursts, and (b) consolidated elasto-plastic response of the film.

those inferred from curvature experiments are not feasible since the resistance of the thin film to indentation, as quantified by $C$, is strongly influenced not only by microplasticity in the form of dislocation bursts, but also by the elastic response of the film. It is also emphasized that the overall elasto-plastic response estimates based on the $P-h$ plots, such as Fig. 4, rely heavily on the extent, frequency and statistical fluctuations of displacement bursts during nano-indentation. As a result, such plots can only be used for relative comparisons of deformation among different film thicknesses, and not for the quantification of yield strength values.

\section{Concluding Remarks}

Nano-indentation experiments have been conducted on polycrystalline thin films of copper, 300, 600 or $1000 \mathrm{~nm}$ in thickness, on silicon substrates. The load-displacement curves exhibit periodic bursts in indentor penetration depth, which is interpreted chiefly to be a consequence of the nucleation of dislocations. The first departure from elastic deformation appears to occur when the maximum shear

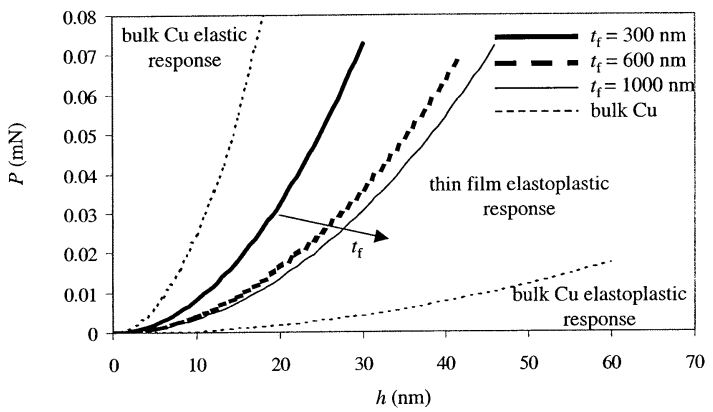

Figure 4. Relative variations in the resistance to indentation, which signifies the resistance to elastoplastic deformation, as a function of film thickness for the $\mathrm{Cu}$ films. 
stress at the tip of the indentor reaches the theoretical shear strength of copper, and is, as anticipated, essentially independent of film thickness. The bursts of dislocation nucleation, occurring at approximately constant indentation loads, are separated by an elastic deformation response which conforms to Kick's law for the sharp Berkovich indentor and which can be estimated solely from the elastic properties of the film. The overall elastoplastic response of the film to nano-indentation, which comprises elastic deformation of the film and microplasticity in the film arising from dislocation nucleation, appears to be sensitive to the film thickness in that the resistance to nano-indentation systematically decreases with increasing film thickness. This trend is fully consistent with curvature measurements on film-substrate systems which reveal that the 'average' yield strength of the thin film increases with decreasing film thickness.

\section{$\underline{\text { Acknowledgment }}$}

This work was supported by the U.S. Department of Energy under Grant W-7405-Eng-48 to the Lawrence Livermore National Laboratory. The authors are grateful to S. Seel and C.V. Thompson for providing the copper thin films used in this study. SS acknowledges helpful discussions with A.E. Giannakopoulos, A. Gouldstone and J.P. Hirth.

\section{$\underline{\text { References }}$}

1. W. C. Oliver and G. M. Pharr, J. Mater. Res. 7, 1564 (1992).

2. S. Suresh and A. E. Giannakopoulos, Acta Mater. 46, 5755 (1998).

3. A. Bolshakov, W. C. Oliver, and G. M. Pharr, J. Mater. Res. 11, 760 (1996).

4. S. G. Corcoran, R. J. Colton, E. T. Lilleodden, and W. W. Gerberich, Phys. Rev. B. 55, 16057 (1997).

5. T. W. Wu, C. Hwang, J. Lo, and P. S. Alexopoulos, Thin Solid Films. 166, 299 (1988).

6. W. Zielinski, H. Huang, S. Venkatraman, and W. W. Gerberich, Phil. Mag. A. 72, 1221 (1995).

7. A. Gouldstone, A. E. Giannakopoulos, S. Suresh, and K.-Y. Zeng, Acta Mater. (1999), submitted.

8. P.-L. Larsson, A. E. Giannakopoulos, E. Soderlund, D. J. Rowcliffe, and R. Vestergaard, Intl. J. Solids Struct. 33, 221 (1996).

9. Q. Ma and D. R. Clarke, J. Mater. Res. 10, 853 (1995).

10. R. Venkatraman, J. C. Bravman, W. D. Nix, P. W. Davies, P. A. Flinn, and D. B. Fraser, J. Electronic Mater. 10, 1231 (1990). 